Bol. Soc. Bot. México 53:73-111 (1993)

\title{
Vegetación y listado florístico de la Barranca de Acazónica, Veracruz, México
}

\author{
María Elena Medina Abreo y GonZalo Castillo-Campos ${ }^{1}$
}

\begin{abstract}
RESUMEN. Se presenta un estudio de vegetación en la Barranca de Acazónica, Municipio de Paso de Ovejas, localizado en el centro del estado de Veracruz. Los tipos de vegetación que se reconocieron para la zona incluyen selva baja caducifolia, selva mediana subperennifolia y vegetación riparia; así como las comunidades secundarias. Se describen brevemente cada uno de ellos y, de manera general, se establecen relaciones de los mismos con algunos factores ambientales. Se presenta un mapa escala 1:70000 de la vegetación de la zona, y un perfil esquemático de la misma. Mediante colectas intensivas durante las cuatro estaciones del año se obtuvieron más de 900 números botánicos correspondientes a 840 especies de 126 familias de plantas vasculares.
\end{abstract}

ABSTRACT. A study of the flora and vegetation of the Barranca de Acazónica, located in the central part of the state of Veracruz, was under taken. The vegetation recognized for the region includes tropical deciduos forest, tropical semideciduos forest, riparian vegetation and secondary vegetation. A description of each vegetation type is given, with a brief discussion of environmental characteristics of the zone. A vegetation map of the region (scale 1:70000) is presented, with a schematic vegetation profile. About 840 species are reported in a floristic checklist, representing 126 families of vascular plants.

Se plantea el trabajo teniendo en cuenta la importancia de la vegetación primaria y el ritmo al que la desplaza, la vegetación secundaria, pues es necesario contar con estudios botánicos y ecológicos que permitan conocer las comunidades vegetales existentes, así como proponer la conservación, el uso y manejo adecuado de las mismas.

\footnotetext{
${ }^{1}$ Instituto de Ecología, A.C., Apdo. Postal 63, 91000, Xalapa, Veracruz, México
} 
Este estudio se realizó en el centro del estado de Veracruz, lugar que por su situación geográfica presenta una variedad de condiciones ambientales, lo cual se ve reflejado en la diversidad florística del área.

La presencia de la selva baja caducifolia en el centro del estado de Veracruz ha sido reportada por diversos autores, entre los cuales pueden citarse los siguientes: Miranda y Hernández X, (1963); Pennington y Sarukhán (1968); Cházaro (1977); Gómez-Pompa (1978); Rzedowski (1978); Ortega (1981); Villanueva (1984); Castillo C. (1985); Gutiérrez (1985); Robles (1986) y Acosta (1986). Sin embargo, aparte de estos estudios, no existen trabajos sistemáticos que se refieran concretamente a la zona de estudio o a sus alrededores.

El objetivo de este estudio es elaborar el inventario florístico de esta zona, describir las comunidades vegetales, la elaboraración de un fotomapa y el perfil esquemático de la vegetación.

\section{ÁREA DE ESTUDIO}

La zona cubre un área de $102 \mathrm{~km}$ se localiza en el centro del estado de Veracruz enmarcada por las coordenadas $19^{\circ} 10^{\prime}$ a $19^{\circ} 16^{\prime}$ Latitud Norte, y $96^{\circ} 32^{\prime}$ a $96^{\circ} 46^{\prime}$ Longitud Oeste (fig.1). Fisiográficamente se sitúa entre la Planicie Costera del Golfo de México y las estribaciones del Eje Neovolcánico Transversal, que en el norte corresponden al Macizo de Palma Sola y al oeste, al Cofre de Perote y Pico de Orizaba, SPP (1984).

Uno de los factores que ha permitido la conservación de las comunidades vegetales primarias es la accidentada topografía de la zona, con altitudes de 200 a $650 \mathrm{~m}$ snm. Las formas topográficas presentes están integradas por cantiles de distinta magnitud, mesetas disectadas, valles y pequeños lomeríos.

Para la zona de estudio se determinaron dos tipos de clima: Aw 1 (w)(e)g y Awo"(w)(i')g, de acuerdo con el sistema de clasificación climática de Koeppen, modificado por García (1981). Para ambas estaciones el clima es cálido subhúmedo, con régimen de lluvias de verano. En lo que a humedad se refiere, para Acazónica es intermedio, presentando un P/T (índice de Lang) de 46.4, y para El Coyol, que es el más seco, un $\mathrm{P} / \mathrm{T}$ de 38.6. En esta última, con presencia de canícula (pequeña temporada seca que se presenta en la mitad caliente del año) (figs. 2 y 3 ).

Para las dos estaciones el porcentaje de lluvia invernal es de 2.8 y 2.9 , con una oscilación térmica de $7.2^{\circ} \mathrm{C}$ (extremoso) y $6.3^{\circ} \mathrm{C}$ (poca oscilación), respectivamente. En ambas, el mes más caliente se presenta antes de junio, por lo que la marcha anual de la temperatura es del tipo Ganges $(\mathrm{g})$. Lo interesante de la precipitación, es su distribución a través del año, debido à que se presenta una época lluviosa y una seca bien definidas; lo que determina el aspecto fisonómico de la vegetación.

De acuerdo con López (1981) y SPP (1984), la región está constituída por rocas sedimentarias del Cenozoico, y se encuentran en una asociación de arenisca-con- 


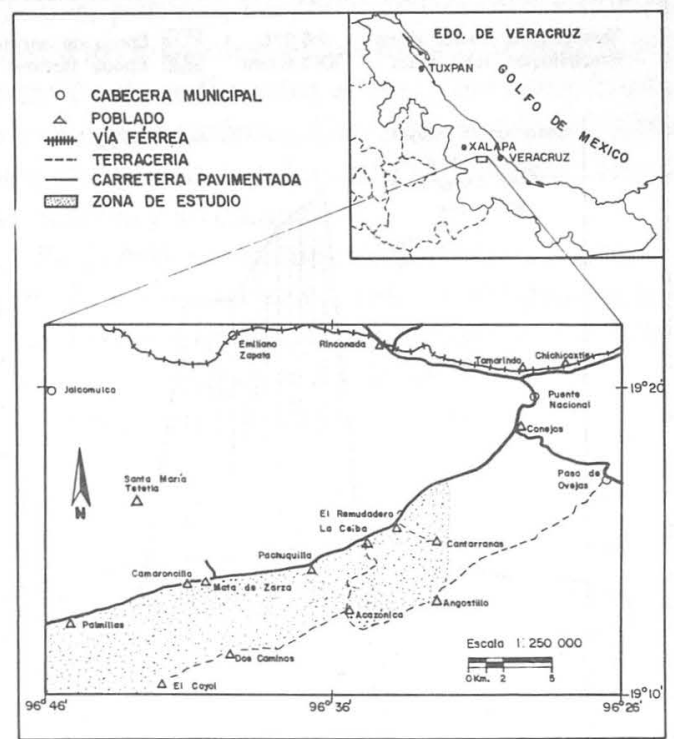

Fig. 1. Localización geográfica de la zona de estudio.

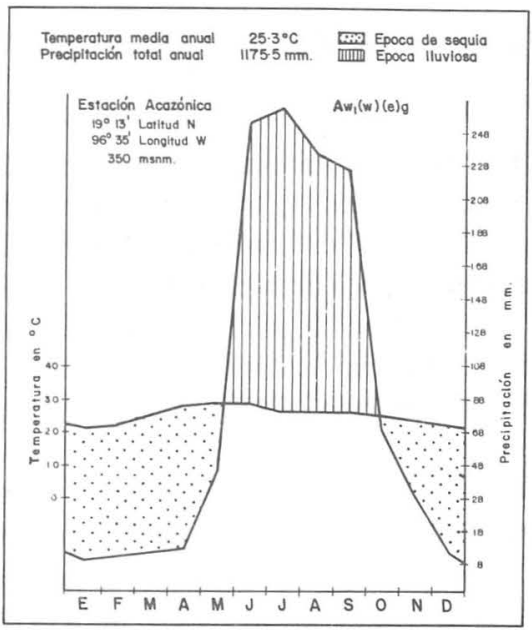

Fig. 2. Diagrama ombrotérmico de Acazónica, Ver. 


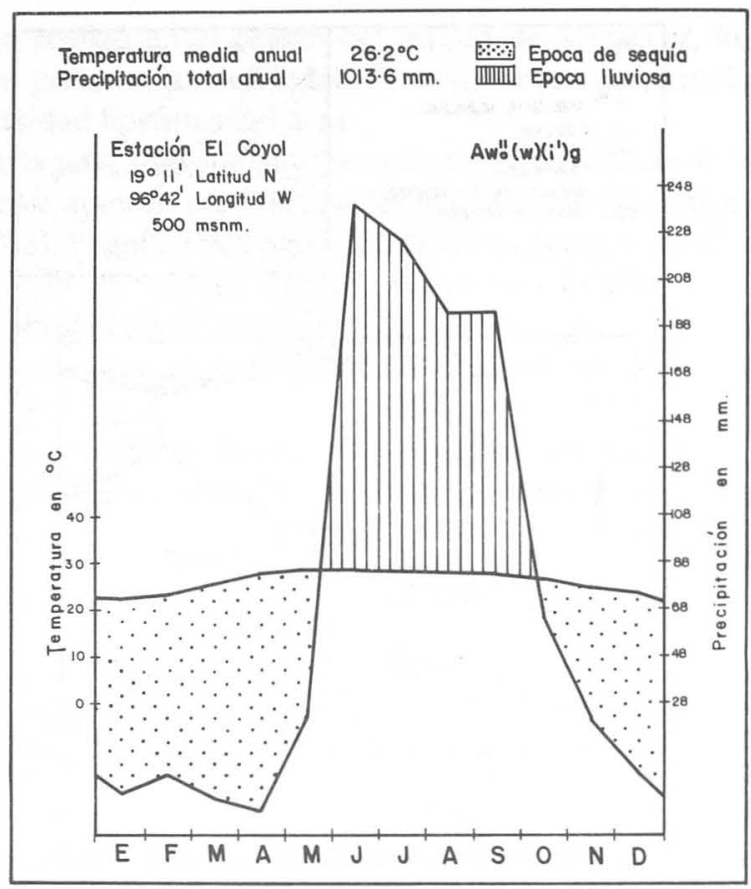

Fig. 3. Diagrama ombrotérmico de El Coyol, Ver.

glomerado, de origen continental. La asociación está formada por clastos redondeados a subredondeados de basalto, andesita, travertino y caliza, en una matriz arenosa (feldespato, cuarzo, plagioclasa, etc) escasamente cementada por carbonatos.

De acuerdo con las observaciones de campo, UACH (1982) y SPP (1984), los suelos que se determinaron de manera general para la zona son: litosoles predominantes en la zona de estudio, caracterizados como suelos someros, pedregosos tanto en la superficie como en el perfil, con drenaje superficial rápido. En las mesetas se encuentran suelos del tipo feozem háplico con profundidad promedio de $15 \mathrm{~cm}$, de color oscuro, con maduración húmica y abundantes raíces, lo que indica que son aptos para la agricultura. Del humus y aumento en el contenido de arcillas del tipo smectitas surgen suelos del tipo vertisol pélico. Son pedregosos, dentro del perfil como en la superficie. Se localizan en las hondonadas de Pachuquilla, y son adecuados para la agricultura.

Otro tipo de suelos presente en la zona son los coluviales. Se forman por el transporte mecánico de sus componentes. Presentan abundantes bloques entremezclados y 
están poco desarrollados, porosos, de color gris oscuro y con materia orgánica. Un último tipo de suelos son los fluvisoles, sin horizontes definidos, pues se forman a partir de la constante aportación de sedimentos a través del agua. Están bien representados en los valles amplios como en Acazónica, Cantarranas y Pachuquilla, donde el río en sus grandes avenidas sale de su cauce y deposita sedimentos. Los fluvisoles son suelos franco-arenosos, permeables y no compactos.

De acuerdo con SPP (1984), la zona de estudio pertenece a la cuenca del río Jamapa, irrigada por una serie de corrientes temporales y permanentes. De estas últimas, la principal, es el río Paso de Ovejas o Chico. Esta es una corriente superficial que muestra fuerte control estructural, puesto que se ha desarrollado sobre una línea de debilidad tectónica o fractura. También existe un nacimiento de aguas termales, al sur de Camaroncillo.

\section{METODOLOGÍA}

En el desarrollo de este trabajo se siguió la metodología propuesta por Miranda et al. (1967). De manera general, las fases fueron:

a) Recorrer la zona para observar y familiarizarse con las principales variantes topográficas, edáficas y florísticas.

b) Con base en las observaciones anteriores se delimitó geográficamente la zona de estudio sobre mapas topográficos escala 1:100000 y con fotografías aéreas escala 1:70000.

c) Teniendo en cuenta la homogeneidad de la zona, el interés de tener representadas la mayoría de las especies de la misma y el hecho de que es necesario colectar las plantas con flor y/o fruto, se realizaron salidas mensuales a los distintos puntos de la zona, por un periódo de 14 meses (enero 1985-febrero 1986). Para cada uno de los ejemplares colectados, se tomaron datos morfológicos, así como del ambiente en que se desarrollan. Los ejemplares están depositados en el herbario XAL y XALU.

d) A través de la interpretación de los elementos de las fotografías aéreas (tono y textura, forma de los objetos, etc) y con base en las observaciones de campo, se delimitó la selva baja caducifolia y las áreas utilizadas para las actividades agropecuarias.

e) El perfil de vegetación se realizó en el sitio más conservado de la Barranca siguiendo una línea transversal que refleja la topografía del lugar, se esquematizaron las especies que representan fisonómicamente la vegetación; complementándose con datos de altitud tomados cada $50 \mathrm{~m}$ (fig.4). 


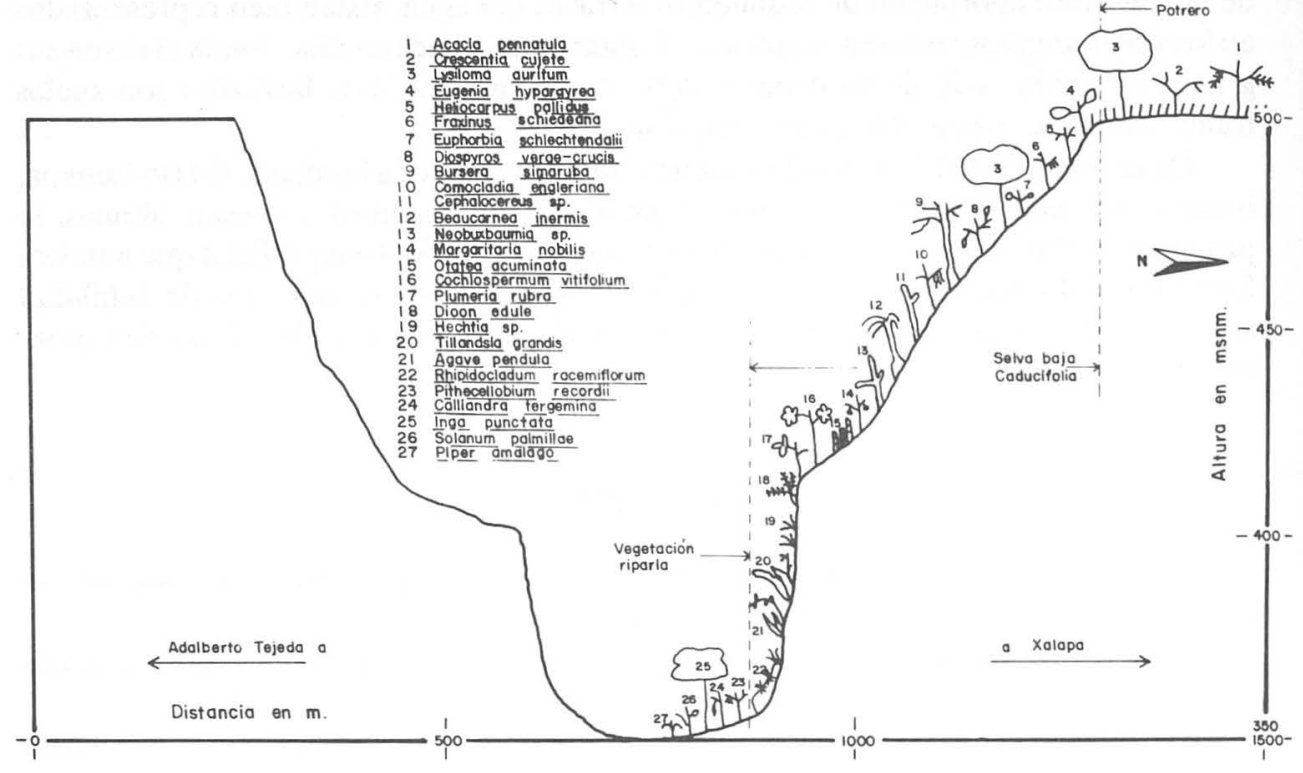

Fig. 4. Perfil esquemático de la vegetación, en la Barranca de Panoaya, en El Coyol, Ver.

\section{RESUlTADOS}

Selva baja caducifolia. Es el tipo de vegetación predominante en la zona, de acuerdo con la clasificación de Miranda y Hernández X. (1963).

Esta vegetación se desarrolla sobre terrenos de ladera, cantiles y mesetas disectadas, donde predominan litosoles, con una asociación de rocas sedimentarias de arenisca-conglomerado.

Durante la época seca la mayoría de los árboles y algunos arbustos se tornan caducifolios, en esta etapa algunos árboles florecen, tal es el caso de Cochlospermum vitifolium, Plumeria rubra, Tabebuia ochracea var. neochrysantha, Pseudobombax ellipticum, entre otros. Durante la época seca, el aspecto de la selva baja caducifolia es desolador y se aprecia un tono gris a diferencia del verde que se observa en la época lluviosa. 
Otra característica de la selva baja caducifolia, es que después de que se ha iniciado la época lluviosa, surgen las plantas anuales, que en su mayoría son hierbas que retoñan o germinan, como Begonia heracleifolia, Begonia peltata, Callisia fragrans, Dorstenia contrajerva y Pseuderanthemum alatum.

En lo que respecta a la estructura de este tipo de vegetación, se distinguen tres estratos: arbóreo, arbustivo y herbáceo.

En el estrato arbóreo, la altura de los árboles varía de 4 a $12 \mathrm{~m}$, y ocasionalmente hasta $15 \mathrm{~m}$. Los miembros más frecuentes de este estrato son Bursera simaruba, Capparis pringlei, Ceiba aesculifolia, Cochlospermum vitifolium, Desmopsis galeottiana, Diospyros oaxacana, Diospyros verae-crucis, Esenbeckia berlandieri, Gyrocarpus jatrophifolius, Jacaratia mexicana y Luehea candida.

En el estrato arbustivo, no es fácil establecer límites de altura, pero generalmente fluctúa de 1.5 a $4 \mathrm{~m}$. Entre las especies más frecuentes se encuentran: Annona globiflora, Bursera fagaroides, Comocladia engleriana, Euphorbia schlechtendalii y Jacquinia aurantiaca. La riqueza de especies del estrato arbustivo, depende de las condiciones ambientales que existen en cada lugar. Otras especies de este estrato son Casearia corymbosa, Colubrina heteroneura, Croton reflexifolius, Erythroxylum mexicanum, Malmea depressa, Podopterus mexicanus, Randia aculeata y Rauvolfia tetraphylla.

El estrato herbáceo normalmente es de $10 \mathrm{~cm}$ a $1 \mathrm{~m}$ de alto, y eventualmente hasta $1.5 \mathrm{~m}$. Este estrato no está bien definido dentro de la selva baja caducifolia. Algunas de las especies que permanecen verdes durante la época seca son Begonia hidrocotyfolia, Begonia manicata, Begonia nelumbiifolia, Justicia breviflora, Peperomia granulosa, Russelia coccinea, Russelia sarmentosa, Salpianthus purpurascens, Scutellaria seleriana, Spigelia anthelmia, Talinum triangulare y Tridax procumbens.

Dentro de la selva baja caducifolia son frecuentes los bejucos que, en ocasiones, llegan a formar enrejados densos y de extensión considerable. Algunas especies de esta forma biológica son Acacia hayesii, Cissus sicyoides, Ipomoea bracteata, Paullinia pinnata, Prestonia mexicana, Rourea glabra y Tetrapteris schiedeana.

En lo que a las epífitas se refiere, no hay gran diversidad. Se encuentran algunos miembros de la familia Orchidaceae y Bromeliaceae, de esta última, el género Tillandsia es el que presennta un mayor número de especies. De las epífitas que se colectaron en la zona, se pueden mencionar: Aechmea bracteata, Encyclia cochleata, E. radiata, Notylia tridachne, Tillandsia balbisiana, T. concolor, T. fasciculata, T. ionantha y T. recurvata.

Es interesante mencionar algunas especies de la selva baja caducifolia, que se desarrollan en microambientes específicos. Este es el caso de Rhipidocladum racemiflorum, gramínea que siempre permanece verde, se desarrolla en sitios húmedos postrándose unos individuos sobre otros; sólo se observó en la barranca de Palmillas. Agave pendula, Dioon edule, Hechtia sp. y Tillandsia grandis, son plantas con hojas en roseta y algo suculentas, se localizan en los cantiles, sobre estrato rocoso, como sucede en la barranca de Palmillas y en la de Panoaya, en El Coyol. 
En sitios muy húmedos, con escasa luz, y sobre sustrato rocoso, se localizan agrupaciones de aráceas, constituídas principalmente por Anthurium podophyllum, Monstera acuminata, Philodendron advena y Syngonium angustatum. Especies suculentas como Agave pendula, Hechtia spp. y Mammillaria sartorii, se desarrollan sobre sustrato rocoso, en pequeños cantiles. Compartiendo este ambiente se encuentran algunas hierbas como Anthurium crassinervium, Begonia peltata y Callisia fragrans. También tiende a ocupar este medio Plumeria rubra.

Es notable la presencia de cactáceas, lo cual refleja las condiciones de escasa humedad dentro de este tipo de vegetación, entre las cuales podemos mencionar a Cephalocereus palmeri var. sartorianus, Cephalocereus sp. y Neobuxbaumia sp. Nopalea dejecta del tipo platiopuntia y los de vida epífita como Hylocereus undatus que constituye masas de varios metros de longitud.

Selva mediana subperennifolia. Este tipo de vegetación se encuentra en los lugares más húmedos con condiciones microclimáticas variadas que, según Gómez-Pompa (1971), permiten a ciertas especies exigentes de humedad vivir en esas zonas, por ejemplo, orillas de ríos, riachuelos y pantanos, etcétera.

El criterio para determinar este tipo de vegetación, se basa en la altura de los árboles que va de 15 a $25 \mathrm{~m}$, al hecho de que alrededor del25-50\% de sus componentes arbóreos pierden sus hojas en lo más acentuado de la época seca. Puesto que no es posible definirla con base en sus componentes florísticos, debido a que, como menciona Gómez-Pompa (1978), son tipos intermedios, compuestos por especies tolerantes a la sequía en las selvas altas y representantes de zonas más húmedas de las selvas bajas, además de algunas especies que solamente crecen en este tipo de vegetación.

Los principales componentes son árboles, de los cuales se pueden mencionar Aphananthe monoica, Aspidosperma megalocarpon, Brosimum alicastrum, Bursera simaruba, Diospyros digyna, Manilkara zapota y Protium copal. Es necesario hacer notar que este tipo de vegetación es poco representativo en la zona de estudio. Sin embargo, su presencia no puede pasar inadvertida en las barrancas húmedas del centro del estado de Veracruz, teniendo en cuenta que este tipo de vegetación se distribuye hacia el norte y sur del mismo.

Vegetación riparia. Aunque esta no ha sido considerada como un tipo de vegetación por Miranda y Hernández X. (1963), en este trabajo se considera como tal de acuerdo con el criterio de Rzedowski (1978). Es necesario hacer notar que este tipo de vegetación es evidente en la zona de estudio. Se distribuye a lo largo de las corrientes de agua permanentes.

La vegetación riparia es muy heterogénea, su composición florística es variable, dependiendo de la influencia de los factores ambientales. De manera general, pueden distinguirse los tres estratos, arbóreo, arbustivo y herbáceo.

La altura del estrato arbóreo frecuentemente es de 3 a $12 \mathrm{~m}$. Constituído por especies como Amphitecna apiculata, Astianthus viminalis, Coussapoa purpusii, Syzygium jambos, Ficus insipida, Lonchocarpus liteomaculatus y Salix humboldtiana. 
El estrato arbustivo, en la mayoría de los casos varía de 1 a $3 \mathrm{~m}$. Especies características de este estrato son Acacia farnesiana, Alibertia edulis, Calliandra tergemina, Icacorea compressa, Inga punctata, Lindenia rivalis, Pithecellobium recordii, Salix taxifolia, Solanum palmillae y Tecoma stans.

El estrato herbáceo es variable, su altura generalmente es de 5 a $60 \mathrm{~cm}$ o más. Algunos de sus componentes son Bletia aff. purpurea, Centradenia inaequilateralis, Cuphea hyssopifolia, Hydrocotile umbellata, Jussiaea suffruticosa y Polygonum longiocreatum.

Ipomoea hastigera, Mikania cordifolia, Strychnos tabascana y Turbina corymbosa son algunos de los bejucos que se desarrollan en el ambiente ripario.

Comunidades secundarias (acahuales). Se incluye en general bajo esta categoría a las comunidades naturales que se establecen como consecuencia de la destrucción total o parcial de la vegetación primaria o clímax, realizada directamente por el hombre o sus animales domésticos (Rzedowski, 1978). La vegetación secundaria compone, probablemente, la biota más importante de los trópicos húmedos, debido a su abundancia, la notable versatilidad de sus respuestas al disturbio y su posible uso presente y futuro (Gómez-Pompa y Vázquez Yanez, 1976).

El área ocupada por vegetación secundaria es considerable en la zona de estudio (fig.5). Son muchas las especies secundarias, entre las más frecuentes están Abutilon trisulcatum, Acacia macracantha, Acacia pennatula, Asterohyptis stellulata, Bahuinia divaricata, Calea ternifolia, Cassia densiflora, Cosmos bipinnatus, Eupatorium odoratum, Ipomoea bracteata, Guazuma ulmifolia, Mucuna pruriens y Pithecotenium crucigerum. Principales cultivos de la región. Teniendo en cuenta el tipo de clima cálido subhúmedo que prevalece en la zona, es común encontrar cultivos adaptados a esa condición, como Carica papaya (papaya) y Mangifera indica (mango).

Esporádicamente se observan algunos árboles de Tamarindus indicus (tamarindo) y pequeños cultivos de Lycopersicon esculentum (jitomate) y de Cucumis sativus (pepino). También es común encontrar cultivos de Zea mays (maíz) y de Phaseolus vulgaris (frijol).

\section{DISCUSIÓN Y CONCLUSIONES}

Las unidades de vegetación determinadas para la zona de estudio son selva baja caducifolia, selva mediana subperennifolia, vegetación riparia y comunidades secundarias.

La zona de estudio se localiza en un área de transición, tanto desde el punto de vista fisiográfico, como del de vegetación. Así tenemos que del lado oeste, hacia las estribaciones del Eje Neovolcánico Transversal, se encuentran establecidos los encinares y al este (Planicie Costera del Golfo de México), predomina la selva baja caducifolia. 


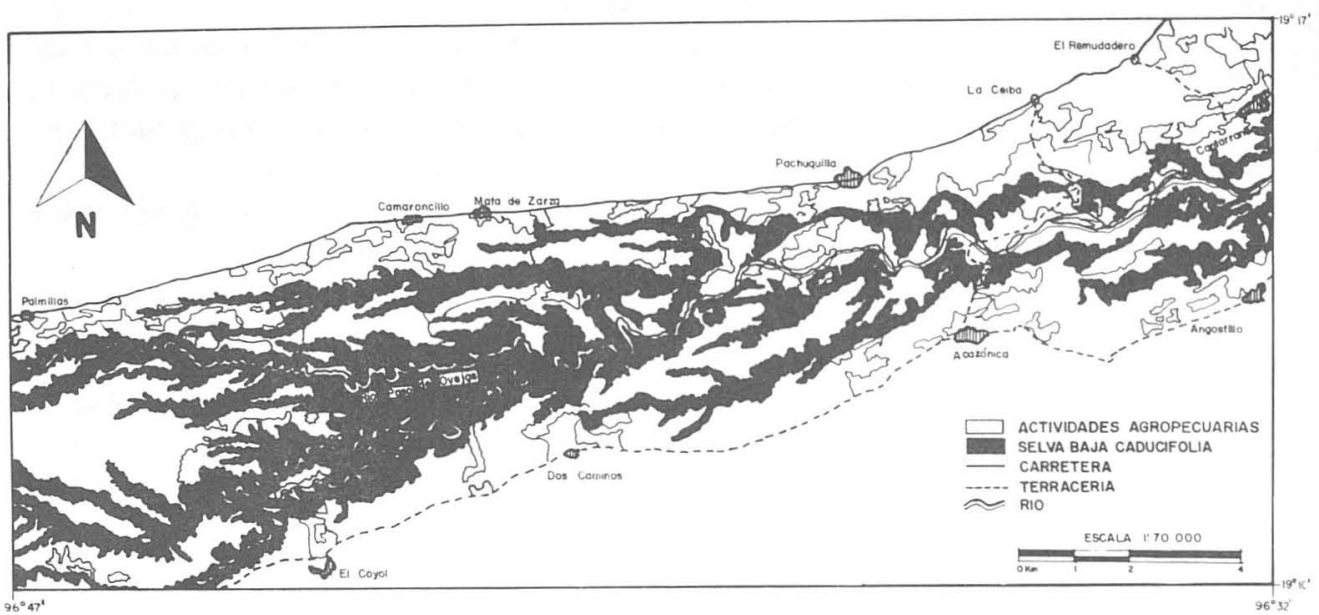

Fig. 5. Distribución de la selva baja caducifolia en la zona de estudio.

Para la zona de estudio se tienen registradas un total de 840 especies, pertenecientes a 126 familias de plantas vasculares. De acuerdo con estos datos, se puede decir, que la zona de estudio es rica florísticamente, pues se encuentran representadas más de la mitad del total de las familias de plantas vasculares reportadas para el estado de Veracruz.

La importancia de realizar trabajos como el presente radica en que se obtienen aportaciones que enriquecen el conocimiento florístico. Algunos ejemplos son los siguientes: Amoreuxia wrightii (Cochlospermaceae), hierba anual en peligro de extinción presente en la zona estudiada o en la Barranca de Acazónica (Vovides, 1982), Bletia stenophylla (Orchidaceae) es un nuevo registro para el estado de Veracruz; Dioscorea cruzensis (Dioscoreaceae), fue colectada por segunda vez después de casi un siglo. Ipomoea bracteata (Convolvulaceae)abundante en la zona, es una especie que no se había registrado de otras partes del estado. 
Lo anterior muestra de manera general, lo escaso que es aún el conocimiento sobre la riqueza florística, y ésto motiva la exploración de aquéllos lugares poco perturbados que aún existen. Los sitios de esa naturaleza, corresponden a barrancas, sierras, cantiles, etc., es decir, lugares donde no es posible realizar actividades agropecuarias o establecer asentamientos humanos.

Afortunadamente en el estado de Veracruz aún existen sitios con esas características, que permiten pensar en la posible conservación de la vegetación primaria.

La riqueza florística de la selva baja caducifolia y las comunidades secundarias es notable. Esto es evidente si se tiene en cuenta que las especies de vegetación secundaria tienden a ocupar una mayor extensión, desplazando a las especies de vegetación primaria. Por otra parte, teniendo en cuenta el área de distribución más restringida de la selva mediana subperennifolia y la vegetación riparia, es lógico que su riqueza florística sea menor que en los casos anteriores.

Es esencial mencionar que, las familias con un mayor número de especies son aquellas que están íntimamente ligadas a las actividades del hombre, ya sea como malezas, alimento o producto del disturbio de ecosistemas. Tal es el caso de las familias Leguminosae, Compositae, Gramineae, Labiatae y Malvaceae, entre otras muchas (Gómez-Pompa, 1983). Al hacer un recuento del número de especies por forma biológica, se observó que la mayor cantidad de árboles y arbustos corresponden a la familia Leguminosae; esto hace pensar que la perturbación en esta zona es evidente. 


\section{Listado florístico de la Barranca de Acazónica, Veracruz}

* Las especies no colectadas por el primer autor, se diferencian por las siguientes iniciales (P) C. A. Purpus, (FV) F. Ventura, (C.C.) Castillo Campos, (J.R.) J. Rzedowski y (SN) plantas registradas para la zona, pero que no fue posible colectar ejemplares herbarios.

** (sb) selva baja caducifolia, (sm) selva mediana subperennifolia, (vr) vegetación riparia, (cs) comunidades secundarias, (A) árbol, (a) arbusto, (b) bejuco y (h) hierba.

\section{ACANTHACEAE}

Aphelandra deppeana Schldl. \& Cham.

No

544

Barleria micans Nees

626

Blechum brownei Juss.

Dyschoriste quadrangularis (Oersted) Kuntze

Elytraria bromoides Oersted

Elytraria imbricata (Vahl) Pers.

Hansteinia aff. gracilis Lindau

Henrya insularis Nees ex Benth.

Jacobinia incana (Nees) Hemsley

FV 9427

Justicia breviflora (Nees) Rusby

Justicia campechiana Standley

Justicia fulvicoma Schldl.

Justicia spicigera Schldl.

Odontonema callistachyum (Schldl. \& Cham. ) Kuntze

Pseuderanthemum alatum (Nees) Radlk.

Ruellia albiflora Fern.

Ruellia inundata Kunth

Ruellia malacosperma Greenman

Ruellia sp.

Siphonoglossa bartletti Standley

Siphonoglossa sessilis (Jacq.) D. Gibson

Stenandrium dulce (Cav.) Nees

sb vr cs sm h a A b

$\mathrm{x}-\mathrm{X}-\mathrm{x} \mathrm{x}-$

$$
-\quad-x-x
$$

$$
-\quad-x-x
$$$$
-\quad-x
$$$$
\mathrm{x}-\mathrm{x}
$$

Tetramerium nervosum Nees

ACHATOCARPACEAE

Achatocarpusmexicanus H. Walter

CC 4311 


\section{ADIANTACEAE}

Adiantumtrapeziforme L.

Adiantum tricholepis Fee

Cheilanthes notholaenoides (Desv.) Maxon exWeath.

Cheilanthes skinneri (Hook.) R. Iryon \& A. Iryon

Hemionitis palmata $\mathrm{L}$

Pteris altissima Poiret

Pteris grandifolia L.

Pteris longifolia $\mathrm{L}$.

\section{AGAVACEAE}

Agave angustifolia Haw.

CC 3543

Agave pendula Schnittsp.

Agave sp.

Yucca sp.

\section{ALISMATACEAE}

Echinodorus andrieuxii (Hook. \& Arn.) Small

FV 2849 AMARANTACEAE

Alternanthera gracilis (Martens \& Galeotti) Loes.

Amaranthus hybridus L.

Amaranthus scariosus Benth.

Amaranthus spinosus $\mathrm{L}$

Gomphrena crassicaulis Brandeg.

Gomphrena serrata L

CC 3555

$\mathrm{SN}$

Gomphrena sp.

Iresine calea (Ibañez) Standley

Iresine celosia $\mathrm{L}$

Iresine diffusa Willd.

Iresine interrupta Benth.

Iresine nigra Uline \& Bray

Pfaffia hookeriana (Hemsley) Greenman

Pfaffia sp.

Pleuropetalum sprucei (Hook. F.) Standley

AMARYLLIDACEAE

Bomarea hirtella (Schldl.) Herbert

Hymenocallis littoralis Salisb.

Zephyranthes carinata Herbert

$\mathrm{CC}$

8871

8665

753

P 8907

3552

718

840

CC 4310

73

886

734

855

ANACARDIACEAE

Astronium fraxinifolium Schott

Comocladia engleriana Loes.

P 8701

Spondiassp. 


\section{ANNONACEAE}

Annona globiflora Schldl.

\begin{tabular}{|c|c|c|c|c|c|c|c|}
\hline 647 & $x$ & - & - & - & - & $\mathrm{x}$ & - \\
\hline 263 & $\mathrm{x}$ & - & - & - & - & - & $\mathrm{x}$ \\
\hline 4321 & $x$ & - & - & - & - & - & $\mathrm{x}$ \\
\hline 842 & $x$ & - & - & - & - & $x$ & $x$ \\
\hline 875 & $x$ & - & - & - & - & $\mathrm{x}$ & - \\
\hline
\end{tabular}

Annona purpurea Mociño \& Sessé

Annona reticulata $\mathrm{L}$.

Desmopsis galeottiana Saff.

Malmea depressa (Baillon) R.E. Fries

\section{APOCYNACEAE}

Aspidosperma megalocarpon Muell. Arg.

Fernaldia pandurata (A. DC.) Woodson

Forsteronia spicata (Jacq.) G. Meyer

Mandevilla donnell-smithii Woodson

Mandevilla subsagittata (Ruíz López \& Pavón) Woodson

Plumeria rubra L.

Prestonia mexicana A. DC.

Rauvolfia tetraphylla $\mathrm{L}$.

Stemmadenia obovata (Hook. \& Arn.) Schumann

Tabernaemontana alba Miller

Thevetia peruviana (Pers.) Schumann

ARACEAE

Anthurium crassinervium (Jacq.) Schott

Anthurium podophyllum (Cham. \& Schldl.) Kunth

Anthurium scandens (Aublet.) Engl.

Monstera acuminata $\mathrm{K}$. Koch

Philodendron advena Schott

Spathiphyllum cochlearispathum (Liebm.) Engl.

Syngonium angustatum Schott

\section{ARALIACEAE}

Dendropanax arboreus (L. ) Decne. \& Planchon

\section{ARISTOLOCHIACEAE}

Aristolochia asclepiadifolia Brandeg

$\begin{array}{rllllllll}68 & \mathrm{x} & - & - & - & \mathrm{x} & - & - & - \\ 114 & \mathrm{x} & - & - & - & \mathrm{x} & - & - & - \\ 4278 & \mathrm{x} & - & - & - & - & - & - & \mathrm{x} \\ 678 & - & - & - & \mathrm{x} & - & - & - & \mathrm{x} \\ 85 & - & \mathrm{x} & - & - & - & - & - & \mathrm{x} \\ 677 & \mathrm{x} & - & - & - & \mathrm{x} & - & - & - \\ 4257 & \mathrm{x} & - & - & - & \mathrm{x} & - & - & -\end{array}$

\section{ASCLEPIADACEAE}

Asclepias curassavica L.

Blepharodon mucronatum (Schldl.) Decne.

Gonolobus sp.

MarsdeniacoulteriHemsley 
Matelearupestris (Brandeg.) Woodson

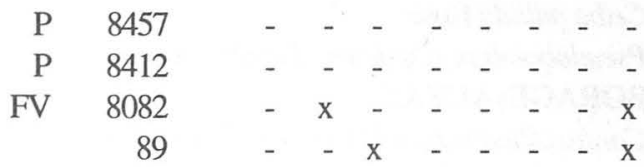

Sarcostemma bilobum Hook. \& Arn.

Sarcostemma clausum (Jacq.) Roem. \& Schultes Sarcostemma elegans Decne.

ASPIDIACEAE

Bolbitis bernoullii (Kuhn exChrist.) Ching

629

ASPLENIACEAE

Asplenium formosum Willd.

661

Asplenium pumilum $\mathrm{Sw}$.

ATHYRIACEAE

Diplazium sp.

CC 4288

BALSAMINACEAE

Impatiens walleriana Hook.

BEGONIACEAE

Begonia heracleifolia Cham. \& Schldl.

Begonia hidrocotylifolia Hook.

Begonia manicata Cels

Begonia nelumbiifolia Cham. \& Schldl.

Begonia peltata Otto \& A. Dietr.

BERBERIDACEAE

Berberis tenuifolia Lindley

\section{BIGNONIACEAE}

Adenocalymma inundatum Mart. exDC.

Amphitecna apiculata A. Gentry

Arrabidaea pubescens (L.) A. Gentry

599

Astianthus viminalis (Kunth) Baillon

Crescentia cujete L.

Cydista aequinoctialis (L.) Miers

Cydista heterophylla Seib.

Melloa quadrivalvis (Jacq.) A. Gentry

Pithecoctenium crucigerum (L.) A. Gentry

FV $\quad 5247$

774

416

472

255

FV 12757

42

FV 12755

CC 3584

487

Stizophyllum riparium (Kunth) Sandw.

Tabebuia ochracea (Cham.) Standl.

var. neochrysantha A. Gentry

Tabebuia rosea (Bertol.) DC

CC $\quad 3597$

Tecoma stans (L.) Juss. exKunth

BLECHNACEAE

Blechnum fragile (Liebm.) Morton \& Lellinger

CC 4287

BOMBACACEAE

Ceibaaesculifolia(Kunth)Britton \& Baker 
Ceiba pallida Rose

Pseudobombax ellipticum (Kunth) Dugand

$\begin{array}{rr}P & 8770 \\ \text { CC } & 4256\end{array}$

BORAGINACEAE

Cordia alliodora (Ruíz López \& Pavón) Oken

Cordia dentata Poiret

Cordia foliosa Martens \& Galeotti

Cordia podocephala Torrey

Cordia pringlei Robinson

Ehretia anacua (Teran \& Berland.) I.M. Johnston

Ehretia tinifolia L.

Heliotropium angiospermum Murray

Heliotropium fruticosum $\mathrm{L}$.

Heliotropium indicum $\mathrm{L}$.

Heliotropium macrostachyum (DC.) Hemsley

$\mathrm{x}$

Heliotropium procumbens Miller

Tournefortia densiflora Martens \& Galeotti

Tournefortia hirsutissima L.

Tournefortia volubilis $\mathrm{L}$.

\begin{tabular}{|c|c|c|c|c|c|c|}
\hline 894 & $x$ & - & $x$ & - & - & $\mathrm{x}$ \\
\hline 3591 & - & - & $x$ & - & - & - \\
\hline 1640 & - & - & - & - & - & - \\
\hline 183 & - & - & $x$ & - & $x$ & - \\
\hline 522 & $x$ & - & $x$ & - & - & $x$ \\
\hline 467 & $x$ & - & - & - & - & - \\
\hline 32 & $\mathrm{x}$ & - & - & - & - & - \\
\hline 733 & - & - & $x$ & - & $x$ & \\
\hline 146 & - & - & $x$ & - & $x$ & - \\
\hline 18 & $x$ & - & - & - & $x$ & - \\
\hline 28 & - & - & $\mathrm{x}$ & - & - & $\mathrm{x}$ \\
\hline 17 & $x$ & - & - & - & $x$ & - \\
\hline 790 & $x$ & - & $x$ & - & $\mathrm{x}$ & - \\
\hline 4293 & - & - & $x$ & - & - & $x$ \\
\hline 59 & - & $\therefore$ & $x$ & - & - & - \\
\hline
\end{tabular}

BROMELIACEAE

Aechmea bracteata (Sw.) Griseb.

CC 4331

Aechmea nudicaulis (L.) Griseb.

Bromelia pinguin $\mathrm{L}$.

4253

Catopsis aff. floribunda L.B. Smith

CC

Hechtia glabra Brandeg.

8506

885

883

Hechtia sp.

Pitcaimia sp.

Tillandsia balbisiana Schultes

Tillandsia concolor L.B. Smith

Tillandsia fasciculata $\mathrm{Sw}$.

Tillandsia grandis Schldl.

Tillandsia gymnobotrya Baker

Tillandsia lucida E. Morren

Tillandsia recurvata $\mathrm{L}$.

Tillandsia schiedeana Steudel

Tillandsia usneoides $\mathrm{L}$. 


\section{BURSERACEAE}

Bursera fagaroides Engl.

Bursera graveolens (Kunth) Triana \& Planchon

264

Bursera simaruba (L.) Sarg.

272

Protium copal (Schldl. \& Cham.) Engl.

\section{BUTOMACEAE}

Limnocharis flava (L.) Buchenau

891

\section{CACTACEAE}

Cephalocereus palmeri Rose var. sartorianus (Rose) Krainz

Cephalocereus sp.

Cephalocereus sp.

Epiphyllum pumilon (Vaupel) Britton \& Rose

Hylocereus undatus (Haw.) Britton \& Rose

Mammillaria sartorii J.A. Purpus

Mammillaria sp.

Neobuxbaumia sp.

Nopalea dejecta (Salm-Dyck) Sd.

\section{CAPPARIDACEAE}

Capparis baducca $\mathrm{L}$.

Capparis pringlei Briq.

Cleome guianensis Aublet

Cleome viscosa $\mathrm{L}$.

Crataeva tapia $\mathrm{L}$.

Morisonia americana $\mathrm{L}$.

\section{CARICACEAE}

Jacaratia mexicana A. DC.

\section{CARYOPHYLLACEAE}

Stellaria cuspidata Willd.

CC $\quad 4269$

\section{CELASTRACEAE}

Elaeodendron trichotomum (Turcz.) Lundell

Maytenus aff. repandus Turcz.

Microtropis schiedeana Loes.

Rhacoma aff. uragoga (Jacq.) Baillon

Rhacoma uragoga (Jacq.) Baillon

Wimmeria concolor Schldl \& Cham. 
Amoreuxiawrightii A. Gray

Cochlospermumvitifolium (Willd.)Sprengel COMBRETACEAE

Combretum fruticosum (Loefl.) Stuntz

COMMELINACEAE

Aploleia monandra (Sw.) Moore

Aploleia multiflora (Martens \& Galeotti) H. Moore

Callisia fragrans (Lindley) Woodson

Campelia zanonia Kunth

Commelina diffusa Burm. F.

Commelina erecta $\mathrm{L}$.

Gibasis schiedeana (Kunth) D. Hunt

Tinantia longipedunculata Standley \& Steyerm.

Tripogandra serrulata (Vahl) Handlos

Zebrina pendula Schnizlein

COMPOSITAE

Ageratina ligustrina (DC.) R. King \& H. Robinson Ageratum houstonianum Miller

Aster bullatus Klatt

Baccharis rhexioides Kunth

Baltimora recta $\mathrm{L}$.

Bidens pilosa $\mathrm{L}$.

Bidens reptans (L.) G. Don

Brickellia diffusa A. Gray

Calea ternifolia Kunth

Calea urticifolia (Miller) DC.

Cosmos bipinnatus Cav.

Cosmos caudatus Kunth

Dahlia coccinea Cav.

Dyssodia aurantia (L.) Robinson

Dyssodia porophylla (Cav.) Cav.

Egletes liebmanni Schultz-Bip.

Elvira biflora DC.

Eupatorium collinum DC.

Eupatorium daleoides (DC.) Hemsley

Eupatoriumodoratum L.

Eupatorium quadrangulare DC.

Eupatorium sp.

Eupatorium sp.
276

CC 3571

CC $\quad 3536$

CC 4291

124

CC 3558

357

725

557

497

587

381

432

CC 4249

869

775

P 8857

FV 6059

559

741

687

486

854

635

FV 7315

508

818

410

FV 9065

FV 2747

CC 4301

680

FV 13809

598

809

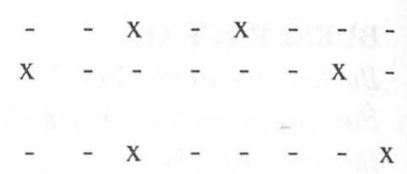

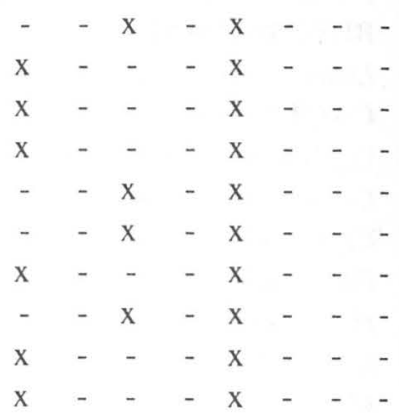

$\mathrm{x}-$

- $x$

$-\quad \mathrm{x}$

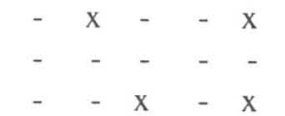

$-\quad-x-x$

$-\quad x-x$

$-\quad-x-x$

$-\quad x-x$

$\mathrm{x}-\mathrm{-}-\mathrm{x}$

$-\quad-x-x$

$-\quad-x-x$

$\mathrm{x}-\mathrm{-}-\mathrm{x}$

$\mathrm{x}-\mathrm{-}-\mathrm{x}$

$-\quad x-x$

$\mathrm{X}-\mathrm{-}-\mathrm{x}$ 
Eupatorium sp.

Eupatorium sp.

Galeana pratensis (Kunth) Rydb.

Isocarpha oppositifolia (L.) R. Br.

Lactuca intybacea Jacq.

Lagascea mollis Cav.

Loxothysanus sinuatus (Less.) Robinson

Melampodium americanum $\mathrm{L}$.

Melampodium divaricatum (Rich.) DC.

Melanthera nivea (L.) Small

Mikania cordifolia (L.F.) Willd.

Mikania houstoniana (L.) Robinson

Mikania micrantha Kunth

Milleria quinqueflora $\mathrm{L}$.

Otopappus curviflorus Hemsley

Parthenium fruticosum Less.

Parthenium hysterophorus L.

Pluchea salicifolia (Miller) Blake

884

155

CC $\quad 4290$

Polymnia maculata Cav.

Porophyllum ruderale (Jacq.) Cass.

Salmea scandens (L.) DC.

$$
\mathrm{x}-\mathrm{x}^{-}-\mathrm{x}-\mathrm{x}
$$

Sclerocarpus divaricatus (Benth. \& Hook.) Hemsley P

Senecio sartorii Schultz-Bip.

Senecio sp.

Stevia ovata Willd.

Stevia sp.

Synedrella nodiflora (L.) Gaertn.

Tithonia tubiformis (Jacq.) Cass.

Tragoceros americanus (Miller) Blake

Tridaxprocumbens $\mathrm{L}$.

Trixis inula Crantz

Verbesina crocata (Cav.) Less.

Verbesina lindenii (Schultz-Bip.) Blake

Verbesina ovolata (Cav.) Less.

VerbesinapersicifoliaDC.

Vernonia deppeana Less.

Vernonia tortuosa (L.) Blake 


\section{CONNARACEAE}

Rourea glabra Kunth

\section{CONVOLVULACEAE}

Evolvulus alsinoides (L.) L. McDonald

391

Evolvulus nummularis (L.) L. McDonald

Ipomoea aff. hederifolia $\mathrm{L}$.

Ipomoea bracteata Cav.

FV $\quad 2748$

Ipomoea hastigera Kunth

722

857

Ipomoea hederifolia $\mathrm{L}$.

671

Ipomoea indica (Burm. F.) Merr.

646

Ipomoea jalapa (L.) Pursh

Ipomoea microsepala Benth.

Ipomoea minutiflora (Martens \& Galeotti) House

FV

7748

FV 7323

6552

Ipomoea setosa Ker Gawler

751

Jacquemontia aff. nodiflora (Desr.) G. Don

Jacquemontia nodiflora (Desr.) G. Don

642

Jacquemontia sphaerostigma (Cav.) Rusby

Merremia aegyptia (L.) Griseb.

675

770

Merremia cissoides (Lam.) Hallier F.

4340

Merremia quinquefolia (L.) Hallier F.

CC

Merremia umbellata (L.) Hallier F.

762

465

763

Turbina corymbosa $(\mathrm{L}$.) Raf.

\section{CRASSULACEAE}

Bryophyllum pinnatum (Lam.) Kurz

814

Echeveria sp.

Sedum hemsleyanum Rose

\section{CUCURBITACEAE}

Cucumis anguria $\mathrm{L}$.

Luffa cylindrica (L.) Roemer

Momordica charantia L.

Polyclathra cucumerina Bertol.

\section{CYPERACEAE}

Cyperusflavus (Vahl) Nees

Cyperus incompletus (Jacq.) L. 
Cyperustenerrimus Presl

Dichromena ciliata Vahl

Eleocharis caribea (Rottb.) Blake

Fuirena simplexVahl

Kyllinga brevifolia Rottb.

Scleria lithosperma Sw.

\section{DIOSCOREACEAE}

Dioscorea convolvulacea Schldl. \& Cham.

Dioscorea cruzensis Knuth

Dioscorea floribunda Martens \& Galeotti

Dioscorea mexicana Scheidw.

DRYOPTERIDACEAE

Tectaria heracleifolia (Willd.) Underw.

\section{EBENACEAE}

Diospyros digyna Jacq.

Diospyros oaxacana Standley

Diospyros verae-crucis Standley

ERYTHROXYLACEAE

Erythroxylum havanense Jacq.

\section{EUPHORBIACEAE}

Acalypha arvensis Poeppig \& Endl.

Acalypha fournieri Muell. Arg.

Acalypha leptopoda Muell. Arg.

Acalypha macrostachya Jacq.

Acalypha oligodonta Muell. Arg.

Acalypha schiedeana Schltr.

Acalypha schlechtendahliana Muell. Arg.

Acalypha subviscida S. Watson

Acalypha unibracteata Muell. Arg.

Acalypha sp.

Adelia barbinervis Cham. \& Schldl.

Argythamnia coatepensis (Brandeg.) Croizat

Argythamnia guatemalensis Muell. Arg.

Argythamnia sitiens (Brandeg.) Ingram

Argythamnia tinctoria Millsp.

Chamaesycehirta(L.) Small

Chamaesyce hissopifolia (L.) Small

Chamaesyce hypericifolia (L.) Millsp.

Chamaesyce thymifolia (L.) Millsp.
445

253

755

754

399

215

643

419

474

389

359

423

868

827

259

782

205

P 9047

349

P 15230

P 9028

368

P 8441

709

493

FV 17242

P 8450

307

P 8844

P 8732

752

184

147

256

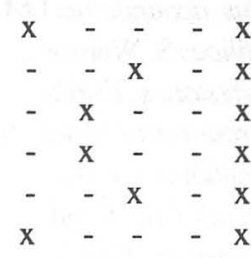

$\mathrm{X}-\mathrm{X}---\mathrm{X}$

$-\quad-X--\quad-X$

$\mathrm{X}-\mathrm{X}-\mathrm{-}-\mathrm{x}$

$\mathrm{x}-\mathrm{-}---\mathrm{x}$

X

$\mathrm{X}$

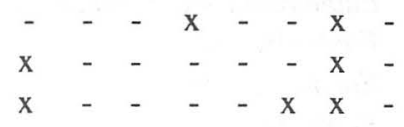

$\mathrm{X}$

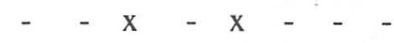

$-\quad-x-x$

$$
\mathrm{x}
$$

x


Cnidoscolus acotinifolius I.M. Johnston

Corythea filipes S. Watson

Croton cortesianus Kunth

P 8713

Croton miradorensis Muell. Arg.

Croton ramillatus Croizat

Croton reflexifolius Kunth

Crotonxalapensis Kunth

Croton sp.

Dalechampia cissifolia Poeppig \& Endl.

Dalechampia heteromorpha Pax \& Hoffman

Dalechampia scandens $\mathrm{L}$.

Euphorbia campestris Cham. \& Schldl.

Euphorbia hirta L.

Euphorbia lancifolia Schltr.

Euphorbia schlechtendalii Boiss.

Euphorbia sp.

Euphorbia sp.

828

Euphorbia sp.

688

Hura polyandra Baillon

289

Jatropha gossypiifolia L.

3596

Jatropha sp.

Manihot aesculifolia (Kunth) Pohl

8448

Margaritaria nobilis L.F.

Pedilanthus calcaratus Schldl.

Pedilanthus sp.

Phyllanthus caroliniensis Walter

Phyllanthus compressus Kunth

Phyllanthus grandifolius L.

Phyllanthus liebmannianus Muell. Arg.

Phyllanthus micrandrus Muell. Arg.

Poinsettia heterophylla Small

Savia sessiflora Willd.

FLACOURTIACEAE

Casearia aculeta Jacq.

Casearia corymbosa Kunth

Casearia obovata Schldl.

Casearia sylvestris Sw. 
Pleuranthodendron lindenii (Turcz.) Sleumer Prockia crucis L.

Xylosma velutinum (Tul.) Triana \& Planchon Zuelania guidonia (Sw.) Britton \& Millsp. Zuelania sp.

\section{GESNERIACEAE}

Achimenes grandiflora (Schiede) DC.

Columnea schiedeana Schltr.

Kohleria spicata (Kunth) Oersted

\section{GRAMINEAE}

Anthephora hermaphrodita (L.) Kuntze

\section{Bambusa sp.}

Bouteloua hirsuta var. glandulosa (Cerv.) Swallen Brachiaria fasciculata (Sw.) L.R. Parodi

Cenchrus brownii Roemer \& Schultes

Dactyloctenium aegyptium (L.) Beauv.

Digitaria ciliaris (Retz.) Koeler

Eragrostiscilianensis (All.)Link

Eragrostis ciliaris (L.) R.Br.

Eragrostis glomerata (Walter) L. Dewey

Eragrostis sp.

Gouinia virgata (Presl) Scribner

Hackelochloa granularis (L.) Kuntze

Heteropogon contortus (L.) Beauv.

Hyparrhenia rufa (Nees) Stapf

Lasiacis aff. divaricata (L.) A. Hitchc.

Lasiacis aff. rugelli (Griseb.) A. Hitchc.

Lasiacis rugelli (Griseb.) A. Hitchc.

Leersia ligularis Trin.

Muhlenbergia minutissima (Steudel) Swallen

Muhlenbergia tenella (Kunth) Trin.

Olyra latifolia L.

Oplismenus aff. hirtellus (L.) Beauv.

Oplismenus rariflorus Presl

FV 10407

$\mathrm{SN}$

P 8473

150

411

334

337

FV $\quad 8805$

FV 8803

P 8696

452

FV 4239

FV 9056

FV 9058

802

175

498

686

603

8474

815

219

681

553

871

Panicum trichoides Sw.

121

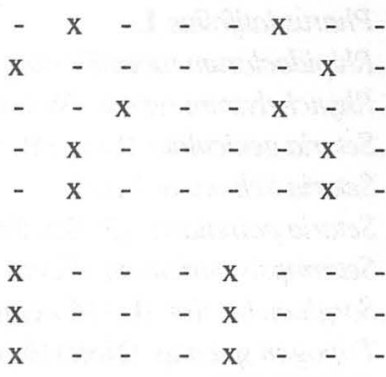

Paspalum langei (Fourn.) Nash 
Pharus latifolius L.

Rhipidoclarum racemiflorum (Steudel) McClure CC 4264 Rhynchelytrum repens (Willd.) C.E. Hubb. Setaria geniculata (Lam.) Beauv. 108

Setaria liebmanni Fourn.

Setaria poiretiana (R. Schultes) Kunth

Setariopsis auriculata (Fourn.) Scribner

Sorghum bicolor (L.) Moench

Tripogon spicatus (Nees) Ekman.

\section{GUTTIFERAE}

Clusia mexicana Vesque.

Clusia quadrangula Bartlett

Rheedia edulis (Seem.) Triana \& Planchon

\section{HELICONIACEAE}

Heliconia schiedeana Klotzsch

CC 4295

HERNANDIACEAE

Gyrocarpus jatrophifolius Domin

\section{HIIPPOCRATEACEAE}

Hippocratea celastroides Kunth

HYDROPHYLLACEAE

Nama biflorum Choisy

Wigandia urens (Ruíz López \& Pavón) Kunth

\section{IRIIDACEAE}

Eustylis purpurea Engelm. \& Gray

Nemastylis sp.

\section{LABIATAE}

Asterohyptis stellulata (Benth) Epling

Hyptis atrorubens Poit.

CC 4324

Hyptis mociniana Benth.

P 14210

Hyptis pectinata (L.) Poit.

P 8704

Hyptis suaveolens Poit.

Hyptis verticellata Jacq.

Ocimum micranthum Willd.

Salvia lasiocephala Benth.

Salvia purpurea Cav.

Salvia riparia Kunth 


\section{LAURACEAE}

Licaria misantlae (Brandegee) Kosterm.

$\begin{array}{rrrrrrrrrr}\mathrm{P} & 8433 & - & - & - & - & - & - & - & - \\ \mathrm{CC} & 4318 & \mathrm{x} & - & - & - & - & \mathrm{x} & - & - \\ & 509 & \mathrm{x} & - & - & - & - & \mathrm{x} & - & - \\ & 555 & \mathrm{x} & - & - & - & - & - & \mathrm{x} & - \\ \mathrm{P} & 14184 & - & - & - & - & - & - & - & -\end{array}$

Nectandra aff. loesenerii $\mathrm{Mez}$

Nectandra sanguinea Rottb.

Nectandra sp.

Phoebe effusa Meissner

\section{LEGUMINOSAE}

Acacia angustissima (Miller) Kuntze

Acacia carbonaria Schltr.

Acacia famesiana (L.) Willd.

Acacia hayesii Benth.

Acacia macracantha Humb. \& Bonpl.

Acacia pennatula (Cham. \& Schldl.) Benth.

Acacia riparia Kunth

Aeschynomene americana $\mathrm{L}$.

Aeschynomene fascicularis Cham. \& Schldl.

Aeschynomene purpusii Brandeg.

Albizia tomentosa (Micheli) Standley

Bauhinia calderonii (Rose) Lundell

Bauhinia cookii Rose

\begin{tabular}{|c|c|c|c|c|c|c|c|c|}
\hline & 705 & - & - & $x$ & - & & & \\
\hline & 563 & - & - & $x$ & - & - & & \\
\hline CC & 3585 & - & $x$ & - & - & - & & \\
\hline & 610 & $x$ & - & - & - & - & & - \\
\hline CC & 3572 & - & - & $x$ & - & - & & \\
\hline & 344 & - & - & $x$ & - & - & & $x$ \\
\hline $\mathrm{P}$ & 16358 & - & - & - & - & - & & - \\
\hline & 636 & - & - & $x$ & - & $x$ & & \\
\hline & 3556 & - & - & $x$ & - & $x$ & & - \\
\hline & 346 & - & - & $x$ & - & - & & - \\
\hline$P$ & 8723 & - & - & - & - & - & & - \\
\hline $\mathrm{P}$ & 11069 & - & - & - & - & - & & - \\
\hline FV & 12760 & - & $x$ & - & - & - & & - \\
\hline & 317 & $x$ & - & $x$ & - & $x$ & & - \\
\hline $\mathrm{FV}$ & 12061 & $x$ & - & - & - & - & & - \\
\hline $\mathrm{CC}$ & 3583 & - & - & $\mathrm{x}$ & - & - & & $x$ \\
\hline $\mathrm{CC}$ & 4342 & - & - & $x$ & - & - & & - \\
\hline $\mathrm{P}$ & 8391 & - & - & - & - & - & & - \\
\hline $\mathrm{P}$ & 8726 & - & - & - & - & - & & - \\
\hline & 286 & - & - & $x$ & - & - & & - \\
\hline $\mathrm{P}$ & 14292 & - & - & - & - & - & & - \\
\hline & 771 & $\mathrm{x}$ & $x$ & - & - & - & & - \\
\hline & 703 & - & - & $x$ & - & - & & - \\
\hline & 615 & $x$ & - & $x$ & - & - & & - \\
\hline & 425 & - & - & $x$ & - & - & & \\
\hline & 479 & - & - & $x$ & - & $x$ & & - \\
\hline P & 8382 & - & - & - & - & - & & - \\
\hline & 575 & - & - & $x$ & - & - & & \\
\hline
\end{tabular}

Bauhinia divaricata $\mathrm{L}$.

Bauhinia jucunda Brandeg.

Caesalpinia cacalaco Humb. \& Bonpl.

Caesalpinia pulcherrima (L.) Sw.

Calliandra canescens (Cham. \& Schldl.) Benth.

Calliandra mexicana Brandeg.

Calliandra rubescens (Martens \& Galeotti) Standley

Calliandra spraguei (Britton \& Rose) Lundell

Calliandra tergemina $(\mathrm{L}$.$) Benth.$

Calopogonium mucunoides Desv.

Canavalia villosa Benth.

Cassia densiflora Martens \& Galeotti

Cassia obtusifolia L.

Cassia oxyphylla Kunth

Centrosema pubescens Benth

Centrosema sagittatum

(Humb. \& Bonpl.) Brandeg. exRiley

Centrosema virginianum (L.) Benth

Chamaecrista nictitans Moench

Cracca aff. mollis Benth.

Cracca ochroleuca (Jacq.) Alef. 
Crotalaria incana $\mathrm{L}$.

Crotalaria nitens Kunth

Crotalaria sagittalis $\mathrm{L}$.

P 8383

Crotalaria vitelina Ker Gawler

Dalbergia amerimnum Benth.

8537

Dalbergia brownei (Jacq.) Urban

Dalbergia glabra (Miller) Standley

5245

Dalea carthaginensis (Jacq.) Macbr.

Dalea cliffortiana Willd.

3563

Dalea diffusa Moric.

490

Dalea foliolosa (Aiton) Barneby

1424

Dalea scopa Barneby

P 15203

Desmodium canum (Gmelin) Schinz \& Thell.

Desmodium helleri Peyr.

Desmodium hirsutum Martens \& Galeotti

Desmodium infractum DC.

12098

Desmodium plicatum Cham. \& Schldl.

Desmodium procumbens A. Hitchc.

Desmodium scorpiurus (Sw.) Desv.

Desmodium tortuosum ( Sw.) DC.

Diphysa minutifolia Rose

Enterolobium cyclocarpum (Jacq.) Griseb.

Erythrina folkersii Krukoff \& Moldenke

Galactiabelizensis Standley

Galactia striata (Jacq.) Urban

Gliricidia sepium (Jacq.) Steudel

Indigofera acasonicae Brandeg.

Indigofera miniata Ortega

Indigofera suffruticosa Miller

Inga punctata Willd.

Lennea melanocarpa (Schltr.) Vatke

Leucaena cruziana Britton \& Rose

Leucaena diversifolia (Schltr.) Benth.

Leucaena purpusii Britton \& Rose

Lonchocarpus dumetorum Brandeg. 
Lonchocarpus fuscopurpureus Brandeg.

Lonchocarpus guatemalensis Benth.

Lonchocarpus liteomaculatus Pittier

Lonchocarpus mexicanus Pittier

Lysiloma auritum (Schldl.) Benth.

Lysiloma divaricata (Jacq.) Macbr.

Machaerium isadelphum (E. Meyer) Amschoff

Macroptilium lathyroides (L.) Urban

Mimosa albida Humb. \& Bonpl. exWilld.

Mimosa ervendbergé A.Gray

Mimosa pudica $\mathrm{L}$.

Mucuna pruriens (L.) DC.

Nissolia fruticosa Jacq.

Pachyrhizus erosus Urban

Phaseolus lathyroides $\mathrm{L}$.

Pithecellobium arboreum (L.) Urban

Pithecellobiumlanceolatum

(Humb. \& Bonpl.) Benth.

Pithecellobium mangense (Jacq.) Macbr.

\begin{tabular}{|c|c|c|c|c|c|c|c|c|}
\hline $\mathrm{P}$ & 8822 & - & - & - & - & & & - \\
\hline $\mathrm{FV}$ & 12759 & - & $\mathrm{x}$ & - & - & - & & $\mathrm{x}$ \\
\hline & 618 & - & $x$ & - & - & - & - & $x$ \\
\hline P & 12036 & - & - & - & - & - & - & - \\
\hline & 564 & - & - & $x$ & - & - & - & $\mathrm{x}$ \\
\hline & 321 & $\mathrm{x}$ & - & - & - & - & - & \\
\hline FV & 8084 & $\mathrm{x}$ & - & - & - & - & $\mathrm{x}$ & - \\
\hline & 478 & - & - & $x$ & - & $\mathrm{x}$ & - & - \\
\hline P & 8385 & - & - & - & - & - & - & - \\
\hline & 235 & $\mathrm{x}$ & - & - & - & - & $\mathrm{x}$ & \\
\hline & 749 & - & - & $\mathrm{x}$ & - & $\mathrm{x}$ & - & \\
\hline & 649 & - & - & $x$ & - & - & - & - \\
\hline $\mathrm{P}$ & 8579 & - & - & - & - & - & - & \\
\hline & 706 & - & - & $x$ & - & - & - & - \\
\hline V & 2850 & - & - & $x$ & - & $\mathrm{x}$ & - & - \\
\hline & 239 & - & & - & - & - & - & \\
\hline
\end{tabular}

Pithecellobium recordii (Britton \& Rose) Standley Rhynchosia caribaea (Jacq.) DC.

Rhynchosia minima (L.) DC.

Senna atonaria (L.) H. Irwin \& Barneby

Senna fruticosa (Miller) H. Irwin \& Barneby

Senna hirsuta (L.) H. Irwin \& Barneby

Senna occidentalis Link

Senna pallida (Vahl) H. Irwin \& Barneby

Senna uniflora (Miller) H. Irwin \& Barneby

Sesbania emerus Urban

Stylosanthes guyanensis $\mathrm{Sw}$.

Stylosanthes humilis Kunth

Tephrosia vicioides Schltr.

Vigna speciosa (Kunth) Verdc.

CC 4248

CC 3593

540

P 8386

161

CC $\quad 3590$

806

747

473

638

464

591

483

P 8894

518

491

260

Zapoteca formosa (Kunth) H. Hernández

Zapotecasp.

494

Zapotecasp.

Zapoteca sp.

Zornia diphylla (L.) Pers. 


\section{LILIACEAE}

Anthericumeleutherandrum (Koch.)H.E.Moore

Beaucarnea inermis (S. Watson) Rose

Smilax aristolochiaefolia Miller

Smilax lanceolata L.

Smilax mollis Humb. \& Bonpl. ex Willd.

$\begin{array}{rrrrrrrrrr} & 514 & \mathrm{x} & - & \mathrm{x} & - & \mathrm{x} & - & - & - \\ \mathrm{SN} & \mathrm{x} & - & - & - & - & - & \mathrm{x} & - \\ \mathrm{P} & 9021 & - & - & - & - & - & - & - & - \\ 266 & \mathrm{x} & - & - & - & - & - & - & \mathrm{x} \\ 653 & \mathrm{x} & - & \mathrm{x} & - & - & - & - & \mathrm{x}\end{array}$

513

519

704

Mentzelia hispida Willd.

\section{LOGANIACEAE}

Buddleia americana $\mathrm{L}$.

Cynoctonum mitreola (L.) Britton

897

P 8920

82

628

Strychnos tabascana Sprague \& Sandw.

\section{LORANTHACEAE}

Phoradendron quadrangulare (Kunth) Krug \& Urban

Phoradendron tamaulipense Trel.

Phradendron sp.

Psittacanthus schiedeanus (Schldl. \& Cham.) Blume ex Schultes

\section{LYTHRACEAE}

Cuphea aff. wrightii A. Gray

Cuphea decandra Aiton

Cuphea hyssopifolia Kunth

Cuphea salicifolia Cham. \& Schldl.

Rotala ramosior (L.) Koehne

\section{MALPIGHIACEAE}

Bunchosia biocellata Schltr.

Bunchosia lanceolata Turcz.

Bunchosia lindeniana Juss.

Byrsonima crassifolia (L.) Kunth

Heteropteris beecheyana Adr. Juss.

402

Hiraea velutina Nied.

Malpighia glabra L.

Mascagnia macroptera (Mociño \& Sessé) Nied.

Stigmaphyllon ellipticum (Kunth) Adr.Juss. 


\section{MALVACEAE}

Abutilon hemsleyanum Rose

Abutilon trisulcatum (Jacq.) Urban

Abutilon sp.

Abutilon sp.

Abutilon sp.

Abutilon sp.

Anoda cristata (L.) Schldl.

Bogenhardia crispa Reichenb.

Gaya minutiflora Rose

Hibiscus brasiliensis L.

Hibiscus purpusii Brandeg.

Hochreutinera amplexifolia (DC.) Fryx.

Malachra fasciata Jacq.

Malvaviscus arboreus Cav.

Pavonia schiedeana Steudel

Robinsonella lindeniana (Turcz.) Rose \& Baker F.

Sida acuta Burm. F.

Sida ciliaris L.

Sida cordifolia L.

Sida glabra Miller

Sida rhombifolia L.

Sida spinosa $\mathrm{L}$.

Sida urens $\mathrm{L}$.

Wissadula amplissima (L.) Fries

Wissadula periplocifolia (L.) Presl

MARANTACEAE

Maranta arundinacea L.

Maranta gibba Smith

MARCGRAVIACEAE

Marcgravia mexicana Gilg

MARTYNIACEAE

Martynia annua $\mathrm{L}$.

\section{MELASTOMATACEAE}

Arthrostemma ciliatum Ruíz López \& Pavón

Centradenia inaequilateralis

(Cham. \& Schldl.) G. Don

Conostegia xalapensis (Bonpl.) D. Don

Miconia laevigata (L.)DC

Miconia sylvatica Schltr.
CC 4271

P 14295

477

794

692

689

CC 3562

481

FV 2749

549

P 14281

P 9007

810

673

CC 4326

227

821

26

62

P 16366

715

22

180

637

735

296

207

74

846

300

594

CC 4251

811

370 


\section{MELIACEAE}

CedrelaodorataL. $\quad 565$

Trichilia hirta L. 845

TrichiliatrifoliaL. 721

\section{MENISPERMACEAE}

Hyperbaena mexicana Miers

841

MOLLUGINACEAE

Mollugo verticilata $\mathrm{L}$.

FV $\quad 8808$

MORACEAE

Brosimum alicastrum $\mathrm{Sw}$.

Cecropia obtusifolia Berto

Chlorophora tinctoria (L.) Gaudich.

Coussapoa purpusii Standley

Dorstenia contrjerva $\mathrm{L}$.

Ficus cotinifolia Kunth

Ficus goldmanii Standley

832

872

Ficus insipida Willd.

Ficus involuta (Liebm.) Miq.

Ficus pertusa L.

8734

880

Ficus sp.

792

Ficus sp.

831

Ficus sp.

663

537

MYRSINACEAE

Ardisia compressa Kunth

Icacorea compressa (Kunth) Standley

100

Parathesis brevipes Lundell

608

MYRTACEAE

Calyptranthes schiedeana O. Berg

Eugenia aff. capuli (Cham. \& Schldl.) O. Berg

Eugenia aff. rhombea (O. Berg) Krug \& Urban exUrban

Eugenia capuli (Cham. \& Schldl.) O. Berg

Eugenia hypargyrea Standley

Eugenia oerstedeana O. Berg

Eugenia rhombea (O. Berg) Krug \& Urban exUrban
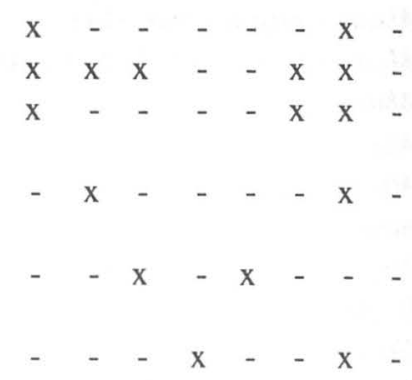

$$
\begin{array}{ll}
- & - \\
\text { X } & -
\end{array}
$$$$
\mathrm{X}
$$$$
\mathrm{X}
$$$$
\text { - } \mathrm{x}
$$$$
-\quad x
$$ 
Eugenia sp.

\begin{tabular}{|c|c|c|c|c|c|c|c|c|}
\hline \multirow{4}{*}{ P } & 93 & - & $x$ & - & - & - & $\mathrm{x}$ & \\
\hline & 16350 & - & - & - & - & - & - & - \\
\hline & 187 & $x$ & - & - & - & - & - & $x$ \\
\hline & 588 & $x$ & - & - & - & - & - & $x$ \\
\hline \multirow[t]{5}{*}{$\mathrm{CC}$} & 4279 & - & $x$ & - & - & - & - & $\mathrm{x}$ \\
\hline & 569 & - & - & $x$ & - & $\mathrm{x}$ & - & - \\
\hline & 320 & - & - & $\mathrm{x}$ & - & $\mathrm{x}$ & - & - \\
\hline & 310 & $\mathrm{x}$ & - & - & - & - & $\mathrm{x}$ & - \\
\hline & 805 & $x$ & - & $x$ & - & $\mathrm{x}$ & - & - \\
\hline \multirow[t]{3}{*}{$\mathrm{P}$} & 6170 & - & - & - & - & - & - & - \\
\hline & 31 & - & - & $x$ & - & - & - & $x$ \\
\hline & 244 & $x$ & - & - & - & $\mathrm{x}$ & - & \\
\hline
\end{tabular}

Myrcianthes fragrans (Sw.) McVaugh

Psidium guajava $\mathrm{L}$.

Psidium sartorianum (O. Berg) Niedenzu

Syzygium jambos (L.) Alston

NYCTAGINACEAE

Boerhavia coccinea Miller

Boerhavia erecta L.

Neea tenuis. Standley

Salpianthus purpurascens (Cav. exLagasca) Hook. \& Arn.

\section{OLACACEAE}

Schoepfia parvifolia Planchon

Schoepfia schreberi Gmelin

\section{OLEANDRACEAE}

Nephrolepis pectinata (Willd.) Schott

\section{ONAGRACEAE}

Jussiaea suffruticosa $\mathrm{L}$.

Oenothera kunthiana (Spach) Munz

OPILIACEAE

Agonandra obtusifolia Standley

ORCHIDACEAE

Bletia stenophylla Schltr.

Catasetum sp.

Encyclia cochleata (L.) Lemée

Encyclia radiata (Lindley ) Dressler

Encyclia sp.

Epidendrum polyanthum Lindley

Habenaria sp.

Ionopsis utricularioides (Sw.) Lindley

Maxillaria densa Lindley

Oncidium carthagenense (Jacq.) Sw.

Oncidium sphacelatum Lindley

OXALIDACEAE

Biophytum dendroides (Kunth) DC.

CC 4298

Oxalis acuminata Cham. \& Schldl.

CC 4270

Oxalis corniculata $\mathrm{L}$.

$\mathrm{CC}$ 


\section{PALMAE}

Chamaedorea elegans Mart.

Chamaedorea tepejilote Liebm. ex Mart.

PAPAVERACEAE

Argemone ochroleuca Sweet

PASSIFLORACEAE

Passiflora filipes Benth.

Passiflora foetida L.

Passiflora foetida L. var. hastata (Bertol.) Masters

Passiflora holosericea L.

Passiflora serratifolia $\mathrm{L}$.

Passiflora suberosa L.

\section{PHYTOLACCACEAE}

Petiveria alliacea $\mathrm{L}$.

Rivina humilis L.

Stegnosperma cubense A. Rich.

\section{PIPERACEAE}

Peperomia asarifolia Cham. \& Schldl.

Peperomia blanda Kunth

Peperomia crassiuscula Mill sp.

Peperomia granulosa Trel.

Peperomia obtusifolia (L.) A. Dietr.

Peperomia sp.

Peperomia sp.

Piper aduncum L.

Piper amalago $\mathrm{L}$.

Piper hispidum Sw.

Piper schiedeanum Steudel

Piper sp.

Pothomorphe umbellata (L.) Miq.

PLUMBAGINACEAE

Plumbago scandens L.

279

POLEMONIACEAE

Loeselia ciliata L.

Loeselia glandulosa (Cav.) G. Don

POLYGALACEAE

Polygala sp.

Polygala sp.
CC 4332

356

226

786

458

631

736

567

326

50

495

350

662

CC 4343

851

640

765

81

231

358

695

367

672

\begin{tabular}{|c|c|c|c|c|c|c|c|}
\hline CC 4285 & $\mathrm{x}$ & - & - & - & $\mathrm{x}$ & - & \\
\hline 75 & - & $\mathrm{x}$ & - & - & - & $\mathrm{x}$ &.- \\
\hline 4332 & - & - & $x$ & - & $\mathrm{x}$ & - & - \\
\hline 356 & $\mathrm{x}$ & - & - & - & - & - & - \\
\hline 226 & - & - & $x$ & - & - & - & - \\
\hline 786 & - & - & $x$ & - & - & - & - \\
\hline 458 & $x$ & - & - & - & - & - & - \\
\hline 631 & - & - & $x$ & - & - & - & - \\
\hline 736 & - & - & $\mathrm{x}$ & - & _- & - & \\
\hline
\end{tabular}

$$
\begin{array}{lll}
x & - & x \\
- & - & x \\
- & x & -
\end{array}
$$

$$
\mathrm{x}
$$

$$
\mathrm{x}
$$$$
\mathrm{x}
$$$$
\mathrm{x}
$$$$
\mathrm{X}
$$$$
\text { - } x
$$$$
\text { - } x
$$

\section{.}

4263 


\section{POLYGONACEAE}

Antigonon cinerascens Martens \& Galeotti

$\begin{array}{rrrrrrrrrr}523 & - & - & \mathrm{x} & - & - & - & - & \mathrm{x} \\ & \mathrm{P} & - & - & \mathrm{x} & - & - & - & - & \mathrm{x} \\ 471 & & - & - & - & - & - & - & - & - \\ 8403 & - & - & - & - & - & - & \mathrm{x} & - \\ 130 & \mathrm{x} & - & - & - & - & - \\ 243 & \mathrm{x} & - & - & - & - & \mathrm{x} & \mathrm{x} & - \\ 45 & \mathrm{x} & - & - & - & - & \mathrm{x} & - & - \\ 776 & - & \mathrm{x} & - & - & \mathrm{x} & - & - & -\end{array}$

Antigonon flavescens $\mathrm{S}$. Watson

Antigonon leptopus Hook. \& Arn.

Coccoloba barbadensis Jacq.

Coccoloba liebmanii Lindau

Podopterus mexicanus Humb. \& Bonpl.

Polygonum longiocreatum Bartlett

\section{POLYPODIACEAE}

Microgramma nitida (J. Smith) A.R. Smith

Niphidium crassifolium (L.) Lellinger

Pecluma alfredii (Rosenst.) Price

\section{PONTEDERIACEAE}

Pontederia sagitatta C. Presl

\section{PORTULACACEAE}

Portulaca grandiflora Hook.

Portulaca umbraticola Kunth

Talinum paniculatum (Jacq.) Gaertn.

Talinum triangulare (Jacq.) Willd.

\section{PROTEACEAE}

Roupala montana Aublet

\section{PSILOTACEAE}

Psilotum complanatum $\mathrm{Sw}$.

\section{RAFFLESIACEAE}

Bdallophyton americanum (R. Br.) Harms

Seytanthus bambusorum Liebm.

$\begin{array}{rrrrrrrrrr} & 834 & \mathrm{x} & - & - & - & - & - & - & \mathrm{x} \\ 4304 & \mathrm{x} & - & - & - & \mathrm{x} & - & - & - \\ 428 & \mathrm{x} & - & - & - & \mathrm{x} & - & - & -\end{array}$

CC 3582

\section{RANUNCULACEAE}

Clematis dioica $\mathrm{L}$.

Clematis grossa Benth.

\section{RHAMNACEAE}

Colubrina heteroneura (Griseb.) Standley

Colubrina triflora Brongn. exSweet

Gouania lupuloides (L.) Urban

Gouania polygama (Jacq.) Urban

\section{RUBIACEAE}

Alibertia edulis (Rich.) A. Rich. exDC.

Bouvardia ternifolia (Cav.) Schltr.

Chiococca alba (L.) Hitchc.

Chione mexicana Standley 
Diodia brasiliensis Sprengel

Diodia teres Walter

Exostema mexicanum Gray

676

Faramea occidentalis (L.) A. Rich.

844

Guettarda elliptica Sw.

451

Hamelia patens Jacq.

Houstonia gracilis

140

641

Lindenia rivalis Benth.

Machaonia acuminata Humb. \& Bonpl.

Oldenlandia microtheca (Schldl. \& Cham.) DC.

Psychotria chamissoana Standley

Psychotria erythrocarpa Schldl.

Psychotria limonensis K. Krause

Psychotria microdon (DC.) Urban

Psychotria miradorensis (Oersted) Hemsley

833

Psychotria trichotoma Martens \& Galeotti

Randia aculeata $\mathrm{L}$.

Randia albonervia Brandeg.

Richardia scabra L.

RUTACEAE

Amyris purpusii $\mathrm{P}$. Wilson

Esenbeckia berlandieri Baillon

Stauranthus perforatus Liebm.

Zanthoxylum sp.

SALICACEAE

Salixhumboldtiana Willd.

CC 4309

Salixtaxifolia Kunth

SAPINDACEAE

Cardiospermum halicacabum L.

Exothea copalillo (Schltr.) Radlk.

Paullinia costaricensis Radlk.

FV 14591

P 14166

P 14172

Paullinia fuscescens Kunth

Paullinia pinnata $\mathrm{L}$.

Paullinia tomentosa Jacq.

Sapindus saponaria L.

Serjania brachycarpa Gray

Serjania brachystachya Radlk.

Serjania racemosa Schum. 
Serjania sp.

Serjania sp.

Serjania sp.

Serjania sp.

Thouinidium decandrum (Humb. \& Bonpl.) Radlk.

Urvillea ulmacea Kunth

P 14365

\section{SAPOTACEAE}

Bumelia persimilis Hemsley

Chrysophyllum mexicanum Brandeg. exStandley

Manilkara zapota (L.) Van Royen

Pouteria durlandii (Standley) Baehni

386

\section{SCHIZAEACEAE}

Anemia adiantifolia (L.) Sw.

Anemia munchii Christ

Anemia phyllitidis (L.) Sw.

Lygodium venustum $\mathrm{Sw}$.

\section{SCROPHULARIACEAE}

Angelonia angustifolia Benth.

Bacopa procumbens (Miller) Greenman

Buchnera pusilla Kunth

Capraria biflora $\mathrm{L}$.

Capraria saxifragaefolia Schldl. \& Cham.

Melasma physalodes (D. Don) Melchior

Russelia campechiana Standley

Russelia coccinea (L.) Wettst.

Russelia sarmentosa Jacq.

Schistophragma sp.

Tetranema roseum

(Martens \& Galeotii) Standley \& Steyerm.

\section{SELAGINELLACEAE}

Selaginella extensa Underw.

SIMAROUBACEAE

Picramnia andicola Tul.

Picrasma mexicana Brandeg.

Simarouba glauca DC.

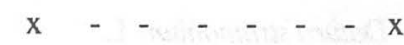

$$
\mathrm{x}--\quad-\quad-x
$$

(n)

$-\quad \mathrm{X}---\mathrm{X}$$$
\mathrm{x}-\mathrm{x}_{-}-\mathrm{-}_{-} \mathrm{-}
$$

$\mathrm{x}$

\section{SOLANACEAE}

Capsicum baccatum $\mathrm{L}$.

Capsicum ciliatum (Kunth) Kuntze

CC 4276

Cestrum dumetorum Schldl. 
Datura stramonium L.

Lycianthes heteroclita (Sendtner) Bitter

Lycianthes rantonnei (Carr. exLescuyer) Bitter

Margaranthus solanaceus Schldl.

Physalis gracilis Miers

Physalis maxima Miller

Physalis pubescens L.

Solanum adscendens Sendtner

Solanum campechiense L.

Solanum candidum Lindley

Solanum diphyllum L.

Solanum hirtum Vahl

Solanum lanceifolium Jacq.

Solanum nudum Dunal

Solanum palmillae Standley

Solanum seaforthianum Andr.

\section{STERCULIACEAE}

Ayenia magna $\mathrm{L}$.

Ayenia micrantha Standley

Ayenia purpusii Brandeg.

Ayenia standleyi Cristobal

Byttneria aculeata Jacq.

Guazuma ulmifolia Lambert

Helicteres guazumaefolia Kunth

Melochia nodiflora Sw.

Melochia pyramidata L.

Melochia tomentosa $\mathrm{L}$.

Waltheria indica $\mathrm{L}$.

\section{THELYPTERIDACEAE}

Thelypteris resinifera (Desv.) Proctor

Thelypteris sp.

\section{THEOPHRASTACEAE}

Jacquinia aurantiaca Aiton

\section{THYMELAEACEAE}

Daphnopsis americana (Miller) J. Johnston

\section{TILIACEAE}

Corchorus orinocensis Kunth

Corchorus siliquosus L.

Heliocarpus americanus L.

\begin{tabular}{|c|c|c|c|c|}
\hline $\mathrm{X}$ & - & $\mathbf{X}$ & - & - \\
\hline - & - & - & $\mathrm{X}$ & - \\
\hline - & - & - & $\mathrm{X}$ & - \\
\hline $\mathbf{X}$ & - & $\mathrm{X}$ & - & - \\
\hline$X$ & - & - & - & - \\
\hline $\mathrm{X}$ & - & $\mathrm{X}$ & - & - \\
\hline - & - & - & - & - \\
\hline 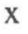 & - & $\mathrm{X}$ & - & - \\
\hline $\mathbf{X}$ & - & $\mathrm{X}$ & - & - \\
\hline - & - & $\mathrm{X}$ & - & - \\
\hline- & - & - & $\mathrm{X}$ & - \\
\hline $\mathrm{X}$ & - & $\mathrm{X}$ & $\mathbf{X}$ & - \\
\hline- & - & - & - & - \\
\hline- & - & - & $\mathrm{X}$ & - \\
\hline - & - & - & $X$ & - \\
\hline $\mathrm{X}$ & - & - & $\mathrm{X}$ & - \\
\hline $\mathrm{X}$ & - & - & $\mathrm{X}$ & - \\
\hline
\end{tabular}

\section{$\mathrm{X}$}

$-$

$\mathrm{X}$

$x-x-x-x$

$-\quad-x$

$-\quad-x$

$\mathrm{x}$

$\mathrm{x}$

$\mathrm{x}$

$\mathrm{x}$ 
Heliocarpus pallidus Rose Luehea candida (DC.) Mart.

Triumfetta dumetorum Schlechter

Triumfetta purpusii Standley

Triumfetta semitriloba Jacq.

\section{TURNERACEAE}

Turnera ulmifolia $\mathrm{L}$.

\section{ULMACEAE}

Aphananthe monoica (Hemsley) Leroy

Celtis caudata Planchon

Celtis iguanaea (Jacq.) Sarg.

\section{UMBELLIFERAE}

Eryngium nasturtiifolium Juss. exF. Delaroche

Hydrocotile bonariensis Lambert

Hydrocotile umbellata $\mathrm{L}$.

\section{URTICACEAE}

Pilea microphylla Liebm.

Pilea pubescens Liebm.

Pouzolzia nivea S. Watson

Pouzolzia sp.

Urera caracasana (Jacq.) Griseb.

\section{VALERIANACEAE}

Valeriana scandens $\mathrm{L}$.

VERBENACEAE

Bouchea prismatica (L.) Kuntze

Callicarpa acuminata Kunth

Citharexylum berlandieri Robinson

Duranta repens $\mathrm{L}$.

Lantana camara $\mathrm{L}$.

Lantana canescens Kunth

Petrea volubilis $\mathrm{L}$.

Priva lappulacea (L.) Pers.

Tamonea curassavica (L.) Pers.

VIOLACEAE

Hybanthus galeotti (Turcz.) Morton

Hybanthus longifolius Melchior

VITACEAE

Ampelocissus erdwendbergii Planchon

Ampelopsis mexicana Rose

$\begin{array}{rrrrrrrrrr} & 826 & \mathrm{x} & - & - & - & - & - & \mathrm{x} & - \\ \mathrm{P} & 169 & \mathrm{x} & - & - & - & - & - & \mathrm{x} & - \\ \mathrm{P} & 11099 & - & - & - & - & - & - & - & - \\ & 9009 & - & - & - & - & - & - & - & - \\ & 808 & \mathrm{x} & - & - & - & \mathrm{x} & \mathrm{x} & - & -\end{array}$

JR 10410

623

879

654

$\begin{array}{ll}\text { P } & 8661\end{array}$

P 8523

756

66

383

201

351

622

878

568

655

315

158

30

548

679

303

152

601

P 8552

165

CC 3537

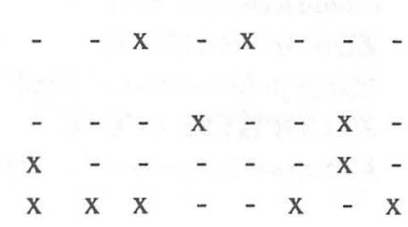

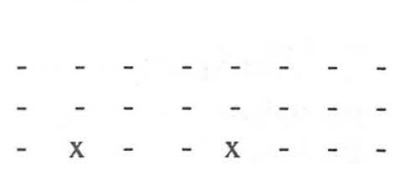

$$
\begin{aligned}
& \mathrm{x} \\
& \mathrm{x} \\
& \mathrm{x} \\
& \mathrm{x} \\
& \mathrm{x}
\end{aligned}
$$
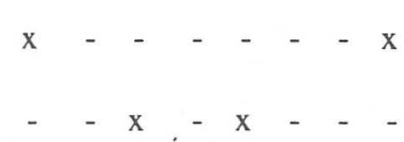$$
\begin{array}{rllll}
- & x & - & x & - \\
x-x & - & - & -
\end{array}
$$$$
\mathrm{x}-
$$$$
\text { - }-x
$$$$
-\quad-x
$$$$
\text { - }-x
$$$$
\mathrm{x}
$$$$
-\quad-x
$$$$
-\quad-
$$ 
Cissus rhombifolia Vahl

Cissus sicyoides $\mathrm{L}$.

ZAMIACEAE

Ceratozamia mexicana Brongn.

Dioon edule Lindley

Zamia loddigesii Miq

ZINGIBERACEAE

Costus pulverulentus C. Presl

ZYGOPHYLLACEAE

Kallstroemia maxima (L.) Torrey \& Gray

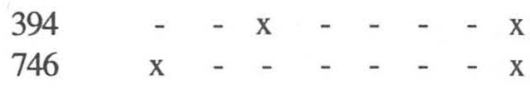

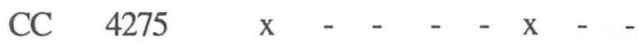

CC $\quad 4261$

234

466

AGRADECIMIENTOS. A la Dra. Margarita Soto Esparza por el apoyo brindado. Al CONACYT, por el financiamiento del proyecto Herbario del Instituto de Ecología, Convenio PCECBNA-021514. Al Dr. Arturo Gómez-Pompa de la Universidad de California, Riverside; Dr. Víctor Rico-Gray, Dr. Andrés Vovides del Instituto de Ecología y Biól. Héctor Narave Flores de la SEDAP, por la revisión del manuscrito.

\section{LITERATURA CITADA}

ACOSTA, P.R. 1986. La vegetación de la sierra de Manuel Díaz, Ver. México. Tesis Facultad de Ciencias Biológicas. UV, Xalapa, Ver.

Castillo C., G. 1985. Integración de paisajes en la región de Jalcomulco, Veracruz. Tesis Facultad de Ciencias Biológicas. UV, Xalapa, Ver.

CHÁZARO B., M. DEJ. 1977. El huizache,Acacia pennatula (Schlecht. \& Cham.) Benth. Especie invasora del centro de Veracruz. Biotica 2(3):1-17.

GARCíA, E. 1981. Modificaciones al sistema de clasificación climática de Koppen. Ed. Offset Larios, México, D.F. 252p.

GÓMEZ-POMPA, A. 1971. Posible papel de la vegetación secundaria en la evolución de la flora tropical. Biotropica 3(2):125-135.

GÓMEZ-POMPA, A.y C. VÁZQUEZ-YÁNES. 1976. Estudios sobre sucesión secundaria en los trópicos cálidos-húmedos: el ciclo de vida de las especies secundarias. En: Gómez-Pompa, A. et al. (Edrs.). Investigaciones sobre la regeneración de selvas altas en Veracruz, México. CECSA, México, D.F. pp. 579-593.

GÓMEZ-POMPA, A. 1978. Ecología de la vegetación del estado de Veracruz. INIREB-CECSA, México, D.F. 91p.

GÓMEZ-POMPA, A. 1983. La destrucción de los ecosistemas tropicales y subtropicales. En: Diez años después de Estocolmo (Desarrollo, Medio Ambiente y Supervivencia). CIFCA, Madrid. pp. 90-106.

GUTIÉRREZ, B.C. 1985. Descripción de la vegetación de la zona inundable de Nevería, Ver. Tesis Facultad de Ciencias Biológicas. UV, Xalapa, Ver.

LópEZ, F. 1981. Geología de México. Ed. Escolar, México, D.F. Tomo III. 446p. 


\section{VEGETACION Y FLORISTICA DE LA BARRANCA DE ACAZONICA, VER.}

Miranda, F. y E. HERnÁNDEZ X. 1963. Los tipos de vegetación de México y su clasificación. Bol. Soc. Bot. México 28:29-179.

MirAndA, F., A. GómEZ-PoMPA y E. HERNÁNDEZ X. 1967. Un método para la investigación ecológica en las regiones tropicales. An. Inst. Biol. Univ. Nal. Autón. México 38(1):101-109.

ORTEGA, O.R. 1981. Vegetación y flora de una corriente de lava (mal país) al NE del Cofre de Perote, Veracruz, México. Biotica 6(1):57-97.

PENNINGTON, T.D. y J. SARUKHÁN. 1963. Manual para la iđentificación de campo de los principales árboles tropicales de México. INIF-FAO. México, D.F., Roma. 413p.

ROBLES, H.L. 1986. La vegetación y uso tradicional de las plantas de la barranca de Monte Rey, Municipio de Axocuapan, Ver., y sus alrededores. Tesis Facultad de Ciencias Biológicas. UV, Xalapa, Ver.

RZEDOWSKI, J. 1978. Vegetación de México. Limusa, México, D.F. 432p.

SPP. 1984. Hoja E 14-3 Veracruz: topográfica, geológica e hidrológica de aguas superficiales. Esc. 1:250000. Dirección General de Geografía del Territorio Nacional. México, D.F.

UACH. 1982. Inventario de áreas erosionadas, rango de pendiente y unidades de suelo del estado de Veracruz. Universidad Autónoma de Chapingo. Texcoco, México. 247p.

VillanUEVA, R. 1984. Plantas de importancia apícola en el ejido de Plan del Río, Veracruz, Mexico. Biotica 9(3):279-340.

VOVIDES, A. 1982. Lista preliminar de plantas mexicanas raras o en peligro de extinción. Biotica 6(2):175188. 